(C) 2010 IEEE. Personal use of this material is permitted. Permission from IEEE must be obtained for all other uses, in any current or future media, including reprinting/republishing this material for advertising or promotional purposes, creating new collective works, for resale or redistribution to servers or lists, or reuse of any copyrighted component of this work in other works. 


\section{Improving N Calculation of the RSI Financial Indicator Using Neural Networks}

\author{
Alejandro Rodríguez-González, Fernando Guldris- \\ Iglesias, Ricardo Colomo-Palacios \\ Computer Science Department \\ Universidad Carlos III de Madrid \\ Leganés, Spain \\ \{alejandro.rodriguez, fernando.guldris, ricardo.colomo\}@uc3m.es
}

\author{
Giner Alor-Hernandez, Ruben Posada-Gomez \\ Computer Science Department \\ Instituto Tecnológico de Orizaba \\ Orizaba, Mexico \\ \{galor,rposada\}@itorizaba.edu.mx
}

\begin{abstract}
Trading and Stock Behavioral Analysis Systems require efficient Artificial Intelligence techniques for analyzing large financial datasets and have become in the current economic landscape a significant challenge for multi disciplinary research. Particularly, Trading oriented Decision Support Systems based on the Chartist or Technical Analysis Relative Strength Indicator (RSI) have been published and used worldwide. However, its combination with Neural Networks as a branch of evolutionary computing which can outperform previous results remain a relevant approach which has not deserved enough attention.

In this paper, we present the Chartist Analysis Platform for Trading (CAST, in short) platform, a proof of concept architecture and implementation of a Trading Decision Support System based on the RSI $N$ value calculation and Feed Forward Neural Networks (FFNN). CAST provides a set of relatively more accurate financial decisions yielded by the combination of Artificial Intelligence techniques to the $\mathrm{N}$ calculation for RSI and a more precise and improved upshot obtained from feed forward algorithms application to stock value datasets.
\end{abstract}

Keywords: neural networks, RSI, trading, prediction

\section{INTRODUCTION}

There has been growing interest in Trading Decision Support Systems in recent years. Forecasting the price movements in stock markets has been a major challenge for common investors, businesses, brokers and speculators. The stock market is considered as a high complex and dynamic system with noisy, non-stationary and chaotic data series [1], and hence, difficult to forecast [2]. However, in spite of its volatility, it is not entirely random [3], instead, it is nonlinear and dynamic [4] or highly complicated and volatile [5]. Stock movement is affected by the mixture of two types of factors [6]: determinant (e.g. gradual strength change between buying side and selling side) and random (e.g. emergent affairs or daily operation variations).

In [1] authors argue the study of stock market is a hot topic, because if successful, the result will transfer to fruitful rewards. Thus, it is obvious that predicting stock market's movement is the long cherished desire of investors, speculators, and industries. However, this market is extremely hard to model with any reasonable accuracy [2]. Prediction of stock price variation is a very difficult task and the price movement behaves more like a random walk and time varying [7].

However, despite the referred complexity, many factors, including macroeconomic variables and stock market technical indicators, have been proven to have a certain level of forecast capability on stock market during a certain period of time. One of the tools for the financial practice is technical analysis, also known as "charting". According to Leigh et al. [8], Charles Dow developed the original Dow Theory for technical analysis in 1884 revisited by Edwards and Magee [9] more than a century before. Technical analysis studies the historical data surrounding price and volume movements of the stock by using charts as the primary tool to forecast future price movements. In recent years, and in spite of several critics (E.g. [10]), technical analysis has proven to be powerful for evaluating stock prices and is widely accepted among financial economists and brokerage firms.

The paper consists of five sections and is structured as follows. Section 2 reviews the relevant literature about technical analysis and its intersection with soft computing. Section 3 discusses the main features of CAST including conceptual model, algorithmic and architecture. Section 4 describes the evaluation of the tool performed. Finally, the paper concludes with a discussion of research findings, limitations and final remarks.

\section{RELATED WORKS}

Stock price prediction using soft intelligence methods is not new. To solve the nonlinear problem and improve stock price evaluation, many researchers have focused on Technical Analysis by using advanced mathematics based techniques [11]. Along with the development of Artificial Intelligence, more researchers have tried to build automatic decision-making systems to predict stock market [12]. Soft computing techniques such as fuzzy logic, neural networks, and probabilistic reasoning draw most attention because of their abilities to handle uncertainty and noise in stock market

In [13] the author was the first to use neural networks for market forecasting in the late 80 's. In the early 90's [14] were used several learning algorithms and prediction methods for developing a prediction system for the Tokyo Stock Exchange Prices Index. In [15] combined the outputs of individual networks using boolean operators to produce a set of composite rules. Other artificial neural networks 
approaches can be found in various works from that decade [16].

As stated before, CAST (Chartist Analysis System For Trading) is based on the use of an improved version of RSI, one of the leading technical analysis indexes. RSI as a part of diverse calculations and formulas is commonly present in the soft computing research [17]. However, using soft computing methods in getting iN calculations is a research task with no presence in the literature. Seeking this new research scenario in this work is proposed CAST, a system that uses generalized feedforward neural network to perform RSI improved calculations.

\section{THE CAST SYSTEM}

Stock price prediction using soft intelligence methods is not new. To solve the nonlinear problem and improve stock price evaluation, many researchers have focused on Technical Analysis by using advanced mathematics based techniques [11]. Along with the development of Artificial Intelligence, more researchers have tried to build automatic decision-making systems to predict stock market [12]. Soft computing techniques such as fuzzy logic, neural networks, and probabilistic reasoning draw most attention because of their abilities to handle uncertainty and noise in stock market.

In [13] the author was the first to use neural networks for market forecasting in the late 80's. In the early 90's [14] were used several learning algorithms and prediction methods for developing a prediction system for the Tokyo Stock Exchange Prices Index. In [15] combined the outputs of individual networks using boolean operators to produce a set of composite rules. Other artificial neural networks approaches can be found in various works from that decade [16].

As stated before, CAST (Chartist Analysis System For Trading) is based on the use of an improved version of RSI, one of the leading technical analysis indexes. RSI as a part of diverse calculations and formulas is commonly present in the soft computing research [17]. However, using soft computing methods in getting iN calculations is a research task with no presence in the literature. Seeking this new research scenario in this work is proposed CAST, a system that uses generalized feedforward neural network to perform RSI improved calculations.

\section{A. Neural Network Module}

The Neural Network Module is the responsible of provide the $\mathrm{N}$ values that are used to calculate RSI and decide if an investor should invest in a certain company. The network used is a generalized feedforward network [19]. The advantage of the generalized Feed Forward (FF) network is the ability to project activities forward by bypassing layers. The result is that the training of the layers closer to the input becomes much more efficient. Hereafter are showed the configuration of the network.

In first place, the input values of the network:

\begin{tabular}{|c|c|}
\hline Input Values & Explanation \\
\hline $\begin{array}{l}\text { IBEX35 Action } \\
\text { Value }\end{array}$ & Value of the market for a concrete day. \\
\hline RSI Optimal & Calculus of optimal RSI using Heuristic \\
\hline N Value & $\mathrm{N}$ value that match with the given RSI Optimal \\
\hline
\end{tabular}

The topology of the network used was divided in an input layer, three hidden layer and an output layer. Table 2 shows the neurons set to each layer.

\begin{tabular}{|lll|llll|}
\hline Layer & $\begin{array}{l}\text { Input } \\
\text { Neurons }\end{array}$ & $\begin{array}{l}\text { Output } \\
\text { Neurons }\end{array}$ & Activation Function & \\
\hline Input & 3 & 15 & Laguarre & $(3$ & Taps, 1 & Tap \\
\hline Hidden & 15 & 10 & TanH & & & \\
\hline Hidden & 10 & 5 & TanH & & & \\
\hline Hidden & 5 & 5 & TanH & & \\
\hline Output & 5 & 1 & Bias & & \\
\hline
\end{tabular}

Table 2 - Neural Network Configuration

The momentum value associated to the layers of the networks was set to 0.7 . Following are showed the training values used in the network:

\begin{tabular}{|c|c|c|}
\hline Number of input data & Cross Validation & Training \\
\hline $\begin{array}{l}4061 \quad(>16 \text { Years of } \\
\text { Financial Data })\end{array}$ & $20 \% \mathrm{o}$ & $\begin{array}{l}10 \% \\
\text { Data }\end{array}$ \\
\hline
\end{tabular}

Table 3 - Neural Network training set

The number of epochs set to train the network was set to 10,000 epochs establishing that the network must stop learning after 500 epochs without improvement in crossvalidation error.

\section{B. Trading System Module}

This module analyzes the result given by neural network module. When a query is formulated to the system, it takes the actual values of the market and builds a query to the neural network. It is important to stand out that in this case the predictions made by the system affect only to the IBEX35 (Spanish) market and not to a concrete company. However, in heuristic section some correction values are shown in order to see that is possible to adapt the heuristic method to make predictions directly to the companies.

The analysis made by the trading system is simple. It takes the value given by the neural network improved $\mathrm{N}$ (iN), generates RSI values following standard calculations and compares it with two extreme values. The comparison yields an output that is considered as follows: 
- If RSI value is higher than 70 the decision that trading system return is a sell signal. This value can be adapted and in some cases the value should be set to 65 instead of 70 .

- If RSI value is lower than 30 the decision that trading system return is a buy signal. This value can be adapted and in some cases the value should be set to 35 instead of 30 .

\section{Heuristic Module}

The heuristic module is in charge of managing the different formulas that provide the heuristic used to generate the optimal values for RSI indicator. As was commented in the previous section exists more than one way to calculate the Optimal RSI value.

The formula of this heuristic is the next:

$R S I=C 1+C 2 * I B E X 35+C 3$

Where:

- IBEX35 represents the current value of the market on the actual date.

- $\mathrm{C} 1$ is a correction parameter set to 206.1103731082200000

- $\mathrm{C} 2$ is a correction parameter set to 0.0213265642264200

- $\mathrm{C} 3$ is a correction parameter set to 0.9947712774802800

The formula has been obtained using statistical techniques applied to the variables involved in the process. Concretely, a linear regression to relate the RSI values with IBEX35 stock market close values has been done.

A total of 30 linear regressions were done to be able to collect possible values of RSI in function of the number of days. Each of this regressions used a different value of RSI when calculate itself with different time intervals. Concretely, it was allowed that the number of days used in the calculation fluctuate between 5 and 35. The reason is collect all the possible values used, because it is not normal that analysts use periods out of this range.

Each of the linear regressions calculated include a parameter called AR(1) to improve the specification of the model. Once results have been obtained, the models that did not fulfill the next requisites were discarded:

- Some of the independent variables is not statistical meaningful for explain the behavior of dependent variable.

- The probability of whole nullity of the model is zero.

- The goodness of fit is not enough good. In this sense, are considerate as no valid the models that cannot explain a $75 \%$ of the variation of the dependent variable.

- The model presents heteroscedasticity [20].
- The model presents autocorrelation.

Finally, in function of the mentioned criterions, has been discarded the models that include the RSI calculated with intervals of days equals to $5,10,12,28,30$ and 35 . With the rest of the models we proceed to calculate a unique equation that is its arithmetic mean, in order that the resulted heuristic is the more representative value of all the valid calculated models.

\section{EVALUATION}

The evaluation of the system consists in two parts:

In the first place, is evaluated how the neural network is able to predict. In more depth, in this evaluation is chosen the best configuration of the neural network set up to calculate better predictions using iN (improved N).

The second part consists of query the neural networks with a certain number of values and verifying the signals that $\mathrm{N}$ value shots to generate a RSI (buy or sell). The aim is to find out the accuracy of CAST.

\section{A. Study 1: Evaluating generalization of neural network}

In the first part, several neural networks were evaluated with different configurations in order to choose the one that showed better results in terms of investment performance. The aim of this study is to find out which neural network configuration provides better results for CAST. Different neural networks configuration schemes were tested and their output was compared with real values in order to choose the one that reached better prediction rates.

Table 4 shows the results from all neural networks tested:

\begin{tabular}{|c|c|c|c|c|}
\hline Name & $\begin{array}{c}\text { \# Input } \\
\text { Parms }\end{array}$ & Parms & Test Ok & $\begin{array}{c}\text { M-Test } \\
\text { Ok (3 } \\
\text { DOC) }\end{array}$ \\
\hline RN1 & 3 & AV, RSI Opt, N & $13.916 \%$ & $\begin{array}{l}60.960 \\
\%\end{array}$ \\
\hline RN2 & 3 & AV, RSI Opt, N & $13.546 \%$ & $\begin{array}{l}66.502 \\
\% \\
\end{array}$ \\
\hline RN3 & 3 & AV, RSI Opt, N & $11.330 \%$ & $\begin{array}{l}64.162 \\
\%\end{array}$ \\
\hline
\end{tabular}

Results show that RN2 configuration provides best results from the set analyzed. Using M-Test Column, results show that RN2 configuration can predict $66.5 \%$ (652 cases from 981 ) in an accurate way, which is a very good approach. With the objective of verifying if results presented statistical significant differences among neural networks configurations, the statistical method analysis of variance ANOVA was used to carry out analysis of variance among groups using the tool SPSS. The level of statistical significance was set at 0.05 . The results of the test indicate that groups present significant differences indicated by the statistical value $(\mathrm{F}(980)=3.247, \mathrm{p}<.05)$. This circumstance implies that, from a statistical point of view, there is a 
significative difference among predictions, and, in other words there are differences in choosing one of them. On the other hand, a t-Student has been calculated between the most accurate neural network (RN2) and the other options. The results shown that there are significant differences between RN1 and RN2 ( $\mathrm{t}(980)=-2.538, \mathrm{p}<.05)$ but aren't significant differences between RN2 and RN3 (t $(980)=--$ $1.091, \mathrm{p}>.05)$, as can be observed from Table 4 taking into account $\mathrm{M}$-Test-OK values. This means that the differences between choosing RN2 and RN3 are just slight.

However, it is important to emphasize if there's a difference between best prediction configuration and real values. This was accomplish using the statistical method Student's t-test (comparison of two means). The results of this test showed significant differences between real values and best predictions corresponding to $\mathrm{RN} 2(\mathrm{t}(980)=-22.238$, $\mathrm{p}<.05)$. This circumstance reveals that there are ways to improve our solution either by better training or using a new setup definition, although it presents undeniable good results. In order to proceed with Study 2 the neural network that is used as reference for further studies is the one named RN2 for its remarkable capability of generalization according to its results.

\section{B. Study 2: Evaluating behavior of neural network in a real case}

In Study 2, once selected in Study 1 the best neural network configuration (RN2), its performance is evaluated in a real market scenario in a given period of time. The aim of this study is to know if iN (improved N) proposed in this paper gives better results than conventional RSI using $\mathrm{N}=14$ days as was suggested by Wilder. In Study 2 this method is noted by RSI14. CAST can be applied to one scenario: whole market prediction (IBEX 35, in this case).

Comparison method is as follows. Given a concrete day and IBEX 35 index, the index is performed (RSI14 and RSI with iN). It advises either "Sell" or "Buy". Once the action is performed the value related to this operation (of selling or buying) is compared with the final value after a period of time (from one day to seven days). If the value is coherent with the prediction (lower if a sell command was send or higher if it was delivered a buy command) then this action increments a success counter for the method proved, RSI14 in this case. Final score is (success operations) / (total operations).

The sample used in this test consisted in the data obtained in the period between 16th December 2005 and 27 th October 2009, a total of 981 values.

The use of periods from 1 to 7 days in evaluation is because the developed system is used to recommend investments in a very short term. E.g. four days means that between buy signal and sell operation the period of time is four days.
Table 5 shows the results of the chosen neural network for RSI with iN prediction compared with RSI14 predicting IBEX 35 behavior:

\begin{tabular}{|l|c|ccc|}
\hline Period & RSI14 & iN & \multicolumn{2}{c|}{ Student's t Test } \\
\hline 1 Day & $45.65 \%$ & $\mathbf{4 8 . 5 7 \%}$ & $(\mathrm{t}(980)$ & $-1.312, \mathrm{p}<0.5)$ \\
\hline 2 Days & $43.00 \%$ & $\mathbf{4 7 . 9 9 \%}$ & $(\mathrm{t}(980)$ & $-2.224, \mathrm{p}<0.5)$ \\
\hline 3 Days & $43.48 \%$ & $\mathbf{4 6 . 3 1 \%}$ & $(\mathrm{t}(980)$ & $-1.271, \mathrm{p}<0.5)$ \\
\hline 4 Days & $42.27 \%$ & $\mathbf{4 6 . 6 7 \%}$ & $(\mathrm{t}(980)$ & $-1.955, \mathrm{p}<0.5)$ \\
\hline 5 Days & $41.79 \%$ & $\mathbf{4 8 . 2 2 \%}$ & $(\mathrm{t}(980)$ & $-2.909, \mathrm{p}<0.5)$ \\
\hline 6 Days & $41.79 \%$ & $\mathbf{4 7 . 1 2 \%}$ & $(\mathrm{t}(980)$ & $-2.411, \mathrm{p}<0.5)$ \\
\hline 7 Days & $42.27 \%$ & $\mathbf{4 8 . 3 1 \%}$ & $(\mathrm{t}(980)$ & $-2.725, \mathrm{p}<0.5)$ \\
\hline & & Table 5 & RSII4 vs iN \\
\hline
\end{tabular}

The results obtained show a better performance of RSI calculated with iN compared to RSI14. All measures presents higher values in iN. On the other hand, only one of the measures by RSI14 present measures above $45 \%$. Higher success values for iN are present in 1 day period $(48.57 \%)$. The first test performed in order to find out if there are significant differences between RSI14 and iN was a Student's t-test (comparison of two means) that is represented in the third column of the table. The tests reveal that exist significant differences between RSI14 and iN results in all the days (from 1 to 7), that in plain words means that iN is significative better that RSI14.

The second issue is to find out if there are significant differences among periods as a whole in iN and RSI15. To do so, it was used One-way ANOVA in order to test for differences among two or more independent groups (in this case seven groups). The result of this test pointed out that there are no significant differences among periods for iN $\mathrm{F}(6867)=0.318, \mathrm{p}>0.5)$ and neither for RSI14 $(\mathrm{F}(6867)=$ $0.749, p>0.5)$, this means that no matter what the period of time is (between one and seven days), the indicator presents values with no significant difference among them. This asseveration can be inferred also from results, showing just slight differences among scores.

The overall conclusion is that, according to results, iN is a more profitable guidance for investors in order to predict IBEX35 stock market.

\section{CONCLUSIONS AND FUTURE WORK}

In the current paper was described a research project about the generation of an improved value (called N) which is the input in RSI formula when a RSI value is going to be calculated. The subjacent idea is the basis for conceiving a system capable of generating in an automatic or semi automatic way investments considering as main invest sector the Spanish IBEX35 stock market. In this paper, the main work is based on the study of cases about the generation of heuristic for a concrete market that allows to generate optimal RSI values for that market taking into account some input parameters, and with that value and some others like the value of the market in the concrete day, the optimal $\mathrm{N}$ calculated from the RSI given by the heuristics or other RSI values use a neural network that allows to generate the 
optimal $\mathrm{N}$ value in the future. With that $\mathrm{N}$ optimal value we are able to generate RSI values based on a certain information and not use always the fixed value $\mathrm{N}=14$.

This paper was based only on the RSI financial indicator and the heuristic methods applied where generated to create a single heuristic formula for the IBEX35 stock market. Taking into account that, two main types of initiatives are considered to be explored in future research. In the first place, our future work plans to generate a heuristic that, with some modifications allow to be used to invest in a company of the stock market. Furthermore, another idea should be the generation of an independent heuristic for each company, without using stock market heuristic. Finally, we will expand the research to investigate broader technical analysis indexes like the MACD (Moving Average Convergence / Divergence) financial indicator or Bollinger Bands.

\section{ACKNOWLEDGMENT}

This work is supported by the Spanish Ministry of Industry, Tourism, and Commerce under the project GODO2 (TSI- 020100-2008-564) and SONAR2 (TSI-020100-2008665), under the PIBES project of the Spanish Committee of Education \& Science (TEC2006-12365-C02-01) and the MID-CBR project of the Spanish Committee of Education \& Science (TIN2006-15140-C03-02). Furthermore, this work is supported by the General Council of Superior Technological Education of Mexico (DGEST). Additionally, this work is sponsored by the National Council of Science and Technology (CONACYT) and the Public Education Secretary (SEP) through PROMEP.

\section{REFERENCES}

[1] Q. Wen, Z. Yang, Y. Song and P. Jia, 'Automatic stock decision support system based on box theory and SVM algorithm', Expert Systems with Applications, 37(2), 1015-1022, (2010).

[2] Y.F. Wang, 'Mining stock prices using fuzzy rough set system', Expert System with Applications, 24(1), 13 23, (2003).

[3]

[4] D.Y. Chiu and P.J. Chen, 'Dynamically exploring internal mechanism of stock market by fuzzy-based support vector machines with high dimension input space and genetic algorithm', Expert Systems with Applications, 36(4), 1240 1248, (2009).
[5] C. Hiemstra and D. Jones, 'Testing for Linear and Nonlinear Granger Causality in the Stock Price-volume Relation', Journal of Finance, 49(5), 1639 1664, (1994).

[6] A.J. Black and D.G. Mcmillan, 'Non-linear predictability of value and growth stocks and economic activity', Journal of Business Finance and Accounting, 31 (3/4), 439 474, (2004).

[7] D. Bao and Z. Yang, 'Intelligent stock trading system by turning point confirming and probabilistic reasoning', Expert Systems with Applications, 34(1), 620 627, (2008).

[8] P.C. Chang and C.H. Liu, 'A TSK type fuzzy rule based system for stock price prediction', Expert Systems with Applications, 34(1), 135 144, (2008).

[9] W. Leigh, N. Modani, R. Purvis, and T. Roberts, 'Stock market trading rule discovery using technical charting heuristics', Expert Systems with Applications, 23(2), 155-159, (2002).

[10] R. Edwards and J. Magee, Technical analysis of stock trends (7th edition). New York: Amacom. (1997).

[11] B.G. Malkiel, A random walk down wall street. New York, NY: Norton \& Co. (1995).

[12] J.L. Wang and S.H. Chan, 'Stock market trading rule discovery using two-layer bias decision tree', Expert Systems with Applications, 30(4), 605-611, (2006).

[13] B. Kovalerchuk and E. Vityaev, Data mining in finance: advances in relational and hybrid methods. Kluwer Academic. (2000).

[14] H. White, H, 'Economic prediction using neural networks: The case of IBM daily stock returns', In Proceedings of the 2nd annual IEEE conference on neural networks, II, 451 458, (1988).

[15] T. Kimoto, K. Asakawa, M. Yoda and M. Takeoka, 'Stock market prediction system with modular neural network', Proceedings of the International Joint Conference on Neural Networks, 1-6, (1990).

[16] R.R. Trippi and D. DeSieno, 'Trading equity index futures with a neural network', Journal of Portfolio Management, 19(1), 27 33, (1992).

[17] M. Aiken, and M. Bsat, 'Forecasting market trends with neural networks', Information Systems Management, 6(4), 42 48, (1994).

[18] J. Yao and J.P. Herbert, 'Financial time-series analysis with rough sets', Applied Soft Computing, 9(3), 1000 1007, (2009).

[19] J.W. Wilder, New Concepts in Technical Trading Systems. Greensboro, NC: Hunter Publishing Company. (1978).

[20] G. Arulampalam and A. Bouzerdoum, 'A generalized feedforward neural network architecture for classification and regression', Neural Networks, 16 (5-6), 561-568, (2003).

[21] R.E. Park, 'Estimation with Heteroscedastic Error Terms', Econometrica, 34, 888, (1966). 